\section{Application of Multiple Scale Analysis on the Parametric Resonance of Continuous Gyroscopic Systems}

\section{Jen-San Chen ${ }^{1}$ and Yu-Ran Lin ${ }^{2}$}

Nayfeh and Mook (1977) presented a multiple scale analysis on the parametric resonance of a non-gyroscopic system with multiple degrees of freedom. This Note extends their method to the case of continuous gyroscopic systems. Orthogonality properties among the eigenfunctions of the unloaded system in the complex state space are used to discretize the equation of motion into a set of first order differential equations. Multiple scale method is then employed to study the parametric resonance of the loaded system up to the second order perturbation. A spinning Rayleigh beam under pulsating axial load is studied as an example.

\section{Equation of Motion}

The equation of motion of a continuous gyroscopic system under parametric excitation may be written as follows,

$M \frac{\partial^{2} w}{\partial t^{2}}+G \frac{\partial w}{\partial t}+K w$

$$
+2 \epsilon \cos \vartheta t\left[\hat{M} \frac{\partial^{2} w}{\partial t^{2}}+\hat{G} \frac{\partial w}{\partial t}+\hat{K} w\right]=0
$$

$M, G$, and $K$ are the mass, gyral, and stiffness operators, respectively, associated with the system free of parametric excitation. $\epsilon$ and $\vartheta$ are the amplitude and frequency of the parametric excitation. $\hat{M}, \hat{G}$, and $\hat{K}$ are operators associated with the periodic excitations. Equation (1) can also be cast in the first-order operator form

$$
(\mathbf{A}+2 \epsilon \cos \vartheta t \hat{\mathbf{A}}) \frac{\partial \mathbf{x}}{\partial t}-(\mathbf{B}+2 \epsilon \cos \vartheta t \hat{\mathbf{B}}) \mathbf{x}=0
$$

by defining the state vector and the matrix differential operators

$$
\begin{aligned}
& \{\mathbf{x} \equiv\} \begin{array}{c}
\frac{\partial w}{\partial t} \\
w
\end{array}, \quad \mathbf{A} \equiv\left[\begin{array}{cc}
M & 0 \\
0 & K
\end{array}\right], \quad \hat{\mathbf{A}} \equiv\left[\begin{array}{cc}
\hat{M} & 0 \\
0 & \hat{K}
\end{array}\right], \\
& \mathbf{B} \equiv\left[\begin{array}{cc}
-G & -K \\
K & 0
\end{array}\right], \quad \hat{\mathbf{B}} \equiv\left[\begin{array}{cc}
-\hat{G} & -\hat{K} \\
\hat{K} & 0
\end{array}\right]
\end{aligned}
$$

The eigenvalues $\lambda_{n}$ of the system without parametric excitation are purely imaginary, and the corresponding eigenfunctions $\mathbf{x}_{n}$ are complex. It has been shown in Chen and Bogy (1992)

\footnotetext{
'Associate Professor

${ }^{2}$ Graduate Student, Department of Mechanical Engineering, National Taiwan University, Taipei, Taiwan 107

Contributed by the Technical Committee on Vibration and Sound for publication in the Journal of Vibration and Acoustics. Manuscript received July 1995; revised Dec. 1995. Associate Technical Editor: S. C. Sinha.
}

that for a gyroscopic system, the orthogonality relations among the eigenfunctions can be written as

$$
\left\langle\mathbf{x}_{n}, \mathbf{A} \mathbf{x}_{p}\right\rangle=0, \quad\left\langle\mathbf{x}_{n}, \mathbf{B} \mathbf{x}_{p}\right\rangle=0 \quad \text { if } \quad \lambda_{n} \neq \lambda_{p}
$$

The inner product between two vectors is defined as $\left\langle\mathbf{x}_{n}, \mathbf{x}_{p}\right\rangle$ $=\int \overrightarrow{\mathbf{x}}_{n}^{T} \mathbf{x}_{p} d V$, where $\overline{\mathbf{x}}_{n}^{T}$ is the transpose of the conjugate of the state vector $\mathbf{x}_{n}$.

We use an expansion in terms of $2 N$ eigenfunctions of the unloaded system to approximate the solution of Eq. (2),

$$
\mathbf{x} \approx \sum_{r=-N}^{N} c_{r}(t) \mathbf{x}_{r}
$$

Substituting Eq. (5) into (2) and taking the inner product between $\mathbf{x}_{n}$ and both sides of Eq. (2), with use of the orthogonality properties (4), we obtain a system of generalized Hill's equations in the first order form,

$$
\frac{d c_{n}}{d t}-i \omega_{n} c_{n}+2 \epsilon \cos \vartheta t \sum_{r=-N}^{N}\left[\hat{A}_{r}^{n} \frac{d c_{r}}{d t}-\hat{B}_{r}^{n} c_{r}\right]=0
$$

where $\omega_{n}$ is the natural frequency of the unloaded system, and

$$
\hat{A}_{r}^{n}=\frac{\left\langle\mathbf{x}_{n}, \hat{\mathbf{A}} \mathbf{x}_{r}\right\rangle}{\left\langle\mathbf{x}_{n}, \mathbf{A} \mathbf{x}_{n}\right\rangle}, \quad \hat{B}_{r}^{n}=\frac{\left\langle\mathbf{x}_{n}, \hat{\mathbf{B}} \mathbf{x}_{r}\right\rangle}{\left\langle\mathbf{x}_{n}, \mathbf{A} \mathbf{x}_{n}\right\rangle}
$$

\section{First Order Multiple Scale Analysis}

The method of multiple scale assumes an expansion of the form

$$
\begin{aligned}
c_{n}(t)=c_{n}^{(0)}\left(T_{0}, T_{1}, T_{2}\right) & +\epsilon c_{n}^{(1)}\left(T_{0}, T_{1}, T_{2}\right) \\
& +\epsilon^{2} c_{n}^{(2)}\left(T_{0}, T_{1}, T_{2}\right)+O\left(\epsilon^{3}\right)
\end{aligned}
$$

where $T_{n} \equiv \epsilon^{n} t$. Substituting (8) into (6) and equating coefficients of like powers of $\epsilon$ yields

$$
\begin{gathered}
\epsilon^{0}: \quad D_{0} c_{n}^{(0)}-i \omega_{n} c_{n}^{(0)}=0 \\
\epsilon^{1}: \quad D_{0} c_{n}^{(1)}-i \omega_{n} c_{n}^{(1)} \\
=-D_{1} c_{n}^{(0)}-2 \cos \vartheta t \sum_{r=-N}^{N}\left[\hat{A}_{r}^{n} D_{0} c_{r}^{(0)}-\hat{B}_{r}^{n} c_{r}^{(0)}\right] \\
\epsilon^{2}: \quad D_{0} c_{n}^{(2)}-i \omega_{n} c_{n}^{(2)}=-D_{2} c_{n}^{(0)}-D_{1} c_{n}^{(1)} \\
-2 \cos \vartheta t \sum_{r=-N}^{N}\left\{\hat{A}_{r}^{n}\left[D_{1} c_{r}^{(0)}+D_{0} c_{r}^{(1)}\right]-\hat{B}_{r}^{n} c_{r}^{(1)}\right\}
\end{gathered}
$$

where $D_{n} \equiv\left(\partial / \partial T_{n}\right)$. The general solution of Eq. (9) can be written in the form $c_{n}^{(0)}=H_{n}\left(T_{1}, T_{2}\right) e^{i \omega_{n} T_{0}}$, and consequently Eq. (10) becomes

$$
\begin{aligned}
D_{0} c_{n}^{(1)}- & i \omega_{n} c_{n}^{(1)}=-D_{1} H_{n} e^{i \omega_{n} T_{0}} \\
& -\sum_{r=-N}^{N} H_{r}\left[i \omega_{r} \hat{A}_{r}^{n}-\hat{B}_{r}^{n}\right]\left[e^{i\left(\omega_{r}+\vartheta\right) T_{0}}+e^{i\left(\omega_{r}-\vartheta\right) T_{0}}\right]
\end{aligned}
$$

Case 1: $\vartheta$ is away from $\omega_{p}+\omega_{q}$ for all possible $p$ and $q$

In this case the secular term is eliminated if $H_{n}=H_{n}\left(T_{2}\right)$, and the particular solution of Eq. (12) can be written as 


$$
\begin{aligned}
c_{n}^{(1)}=-\sum_{r=-N}^{N}\left(i \omega_{r} \hat{A}_{r}^{n}-\hat{B}_{r}^{n}\right) \\
\quad \times\left[\frac{e^{i\left(\omega_{r}+\vartheta\right) T_{0}}}{i\left(\omega_{r}+\vartheta-\omega_{n}\right)}+\frac{e^{i\left(\omega_{r}-\vartheta\right) T_{0}}}{i\left(\omega_{r}-\vartheta-\omega_{n}\right)}\right]
\end{aligned}
$$

Consequently, no unstable vibration will be induced to the first order perturbation.

Case 2: Combination resonance when $\vartheta$ is near $\omega_{p}+\omega_{q}$

In this case the transition curve in the $\vartheta \epsilon$-plane is $\vartheta=\omega_{p}+$ $\omega_{q}+2 \epsilon \sqrt{\Lambda_{q}^{p}}$, where

$$
\Lambda_{q}^{p}=-\left(i \omega_{q} \hat{A}_{q}^{-p}-\hat{B}_{q}^{-p}\right)\left(i \omega_{p} \hat{A}_{-p}^{q}+\hat{B}_{-p}^{q}\right)
$$

In the case when $\operatorname{Im}\left(\Lambda_{q}^{p}\right) \neq 0$, the response is always unstable.

Case 3: Combination resonance when $\vartheta$ is near $\omega_{p}+\omega_{q}$ and $\omega_{s}-\omega_{a}$

In this case we assume that $\vartheta=\omega_{p}+\omega_{q}+\epsilon \sigma_{1}$ and $\vartheta=\omega_{s}$ $-\omega_{q}+\epsilon \sigma_{2}$. The secular terms in Eq. (13) are eliminated if

$$
-\alpha^{2} \beta^{2}+4 \beta^{3}+4 \alpha^{3} \gamma-18 \alpha \beta \gamma+27 \gamma^{2}=0
$$

where $\alpha=\sigma_{2}-\sigma_{1}, \beta=-\sigma_{1} \sigma_{2}+\Lambda_{q}^{p}+\Lambda_{s}^{-q}$, and $\gamma=$ $-\sigma_{1} \Lambda_{s}^{-q}+\sigma_{2} \Lambda_{q}^{p}$.

\section{Second Order Multiple Scale Analysis}

After solving the zeroth and the first order solutions $c_{n}^{(0)}$ and $c_{n}^{(1)}$, the equation for the second order perturbation, Eq. (11), can be written as

$$
\begin{aligned}
& D_{0} c_{n}^{(2)}-i \omega_{n} c_{n}^{(2)}=-D_{2} H_{n} e^{i \omega_{n} T_{0}}
\end{aligned}
$$

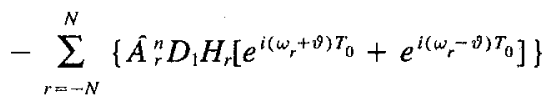

$$
\begin{aligned}
& -\sum_{r=-N}^{N} \sum_{s=-N}^{N} \sum_{p=1}^{2}\left\{H_{s}\left[e^{i\left(\omega_{s}+2(-1)^{p} v\right) T_{0}}+e^{i \omega_{s} T_{0}}\right]\right. \\
& \left.\times\left[\left(i \omega_{s} \hat{A}_{s}^{r}-\hat{B}_{s}^{r}\right)\left(\frac{-\hat{A}_{r}^{n}\left(\omega_{s}+(-1)^{p} \vartheta\right)-i \hat{B}_{r}^{n}}{\omega_{s}+(-1)^{p} \vartheta-\omega_{r}}\right)\right]\right\}
\end{aligned}
$$

Case 1: $\vartheta$ is away from $\omega_{p}+\omega_{q}$ and $2 \vartheta$ is away from $\omega_{l}$ $+\omega_{k}$ for all possible $l$ and $k$

In this case the system is always stable to the second order as long as all $\hat{A}_{n}^{r}$ are real and all $\hat{B}_{n}^{r}$ are purely imaginary.

Case 2: $\vartheta$ is away from $\omega_{p}+\omega_{q}$ and $2 \vartheta$ is near $\omega_{l}+\omega_{k}$ In this case the transition curve is found to be

$$
\vartheta=\frac{\omega_{l}+\omega_{k}}{2}+\epsilon^{2}\left(\frac{i \chi_{k}-i \bar{\chi}_{l}}{2} \pm \sqrt{\bar{\mu}_{k}^{l} \mu_{l}^{k}}\right)
$$

where

$$
\begin{aligned}
& \mu_{l}^{k}=\sum_{r=-N}^{N} {\left[\left(i \omega_{l} \hat{A}_{-l}^{r}+\hat{B}_{-l}^{r}\right)\left(\frac{\hat{A}_{r}^{k}\left(\omega_{l}-\vartheta\right)-i \hat{B}_{r}^{k}}{\omega_{l}-\vartheta+\omega_{r}}\right)\right] } \\
& \chi_{n}=\sum_{r=-N}^{N} \sum_{s=1}^{2}\left\{\left(i \omega_{n} \hat{A}_{n}^{r}-\hat{B}_{n}^{r}\right)\right. \\
&\left.\times\left[\frac{-\hat{A}_{n}^{r}\left(\omega_{n}+(-1)^{s} \vartheta\right)-i \hat{B}_{n}^{r}}{\omega_{n}+(-1)^{s} \vartheta-\omega_{r}}\right]\right\}
\end{aligned}
$$

Case 3: $\vartheta$ is near $\omega_{p}+\omega_{q}$

In this case the transition curve is found to be

$$
\begin{aligned}
\vartheta=\omega_{p}+\omega_{q}+2 \epsilon & \sqrt{\Lambda_{q}^{p}}+\epsilon^{2} i\left[-\bar{\chi}_{q}+\hat{\chi}_{p}-\hat{A}_{p}^{-q} \hat{B}_{-q}^{p}\right. \\
& \left.+\hat{A}_{-q}^{p} \hat{B}_{p}^{-q}-i\left(\omega_{p}+\omega_{q}\right) \hat{A}_{p}^{-q} \hat{A}_{-q}^{p}\right]
\end{aligned}
$$

where

$$
\begin{aligned}
\hat{\chi}_{p}=\sum_{\substack{r=-N \\
r \neq q}}^{N} \sum_{s=1}^{2}\left\{\left(i \omega_{p} \hat{A}_{p}^{r}-\hat{B}_{p}^{r}\right)\right. \\
\\
\left.\quad \times\left[\frac{-\hat{A}_{r}^{p}\left(\omega_{p}+(-1)^{s} \vartheta\right)-i \hat{B}_{r}^{p}}{\omega_{p}+(-1)^{s} \vartheta-\omega_{r}}\right]\right\}
\end{aligned}
$$

Case 4: $\vartheta$ is near $\omega_{p}+\omega_{q}$ and $\omega_{s}-\omega_{q}$

In this case we assume that $\vartheta=\omega_{p}+\omega_{q}+\epsilon \sigma_{1}$ and $\vartheta=\omega_{s}$ $-\omega_{q}+\epsilon \sigma_{2}$. The transition curve is defined by Eq. (15) with the constants $\alpha, \beta$, and $\gamma$ being replaced by

$$
\alpha=-i \epsilon\left(b_{1}+b_{5}+b_{9}\right)-\sigma_{1}+\sigma_{2}
$$

$\beta=b_{2} b_{4}+b_{6} b_{8}-\sigma_{1} \sigma_{2}+i \epsilon\left(b_{5} \sigma_{1}+b_{9} \sigma_{1}-b_{1} \sigma_{2}-b_{5} \sigma_{2}\right)$

$$
+\epsilon^{2}\left(b_{3} b_{7}-b_{1} b_{5}-b_{1} b_{9}-b_{5} b_{9}\right)
$$

$\gamma=b_{2} b_{4} \sigma_{2}-b_{6} b_{8} \sigma_{1}+i \epsilon\left(b_{2} b_{6} b_{7}+b_{3} b_{4} b_{8}-b_{1} b_{6} b_{8}-b_{2} b_{4} b_{9}\right.$

$$
\begin{gathered}
\left.+b_{5} \sigma_{1} \sigma_{2}\right)+\epsilon^{2}\left(b_{5} b_{9} \sigma_{1}-b_{1} b_{5} \sigma_{2}\right)+i \epsilon^{3}\left(b_{1} b_{5} b_{9}-b_{3} b_{5} b_{7}\right) \\
b_{1}=\hat{A}_{q}^{-p}\left(i \omega_{p} \hat{A}_{-p}^{q}+\hat{B}_{-p}^{q}\right)+\hat{\chi}_{p}, \quad b_{2}=i \omega_{q} \hat{A}_{q}^{-p}-\hat{B}_{q}^{-p} \\
b_{3}=-\hat{A}_{q}^{-p}\left(i \omega_{s} \hat{A}_{s}^{q}-\hat{B}_{s}^{q}\right)+\bar{\mu}_{s}^{p}, \quad b_{4}=-\left(i \omega_{p} \hat{A}_{-p}^{q}+\hat{B}_{-p}^{q}\right) \\
b_{5}=-\hat{A}_{-p}^{q}\left(i \omega_{q} \hat{A}_{q}^{-p}-\hat{B}_{q}^{-p}\right)+\hat{\chi}_{q}-\hat{A}_{s}^{q}\left(i \omega_{q} \hat{A}_{q}^{s}-\hat{B}_{q}^{s}\right) \\
b_{6}=i \omega_{s} \hat{A}_{s}^{q}-\hat{B}_{s}^{q}, \quad b_{7}=\hat{A}_{q}^{s}\left(i \omega_{p} \hat{A}_{-p}^{q}+\hat{B}_{-p}^{q}\right)+\mu_{p}^{s} \\
b_{8}=i \omega_{q} \hat{A}_{q}^{s}-\hat{B}_{q}^{s}, \quad b_{9}=-\hat{A}_{q}^{s}\left(i \omega_{s} \hat{A}_{s}^{q}-\hat{B}_{s}^{q}\right)+\hat{\chi}_{s}
\end{gathered}
$$

Case 5: $\vartheta$ is near $\omega_{p}+\omega_{q}$ and $2 \vartheta$ is near $\omega_{s}-\omega_{q}$

In this case we assume that $\vartheta=\omega_{p}+\omega_{q}+\epsilon \sigma_{1}$ and $2 \vartheta=$ $\omega_{s}-\omega_{q}+\epsilon^{2} \sigma_{2}$. The transition curve is defined again by $\mathrm{Eq}$. (15) with the constants being replaced by

$$
\begin{gathered}
\alpha=-i \epsilon\left(b_{1}+b_{4}+b_{7}\right)-\sigma_{1}+\epsilon \sigma_{2} \\
\beta=b_{2} b_{3}-\epsilon \sigma_{1} \sigma_{2}+i \epsilon \sigma_{1}\left(b_{4}+b_{7}\right) \\
+\epsilon^{2}\left(b_{5} b_{6}-b_{1} b_{4}-b_{1} b_{7}-b_{4} b_{7}\right)-i \epsilon^{2} \sigma_{2}\left(b_{1}+b_{4}\right) \\
\gamma=\epsilon\left(b_{2} b_{3} \sigma_{2}-i b_{2} b_{3} b_{7}\right)+\epsilon^{2}\left(b_{4} b_{7} \sigma_{1}-b_{5} b_{6} \sigma_{1}+i b_{4} \sigma_{1} \sigma_{2}\right) \\
+\epsilon^{3}\left(i b_{1} b_{4} b_{7}-i b_{1} b_{5} b_{6}-b_{1} b_{4} \sigma_{2}\right) \\
b_{2}=i \omega_{q} \hat{A}_{q}^{-p}-\hat{B}_{q}^{-p}, \quad b_{3}=-\left(i \omega_{p} \hat{A}_{-p}^{q}+\hat{B}_{-p}^{q}\right), \\
b_{4}=-\hat{A}_{-p}^{q}\left(i \omega_{q} \hat{A}_{q}^{-p}-\hat{B}_{q}^{-p}\right)+\hat{\chi}_{q}, \quad b_{5}=\bar{\mu}_{s}^{-q}, \\
b_{6}=\mu_{-q}^{s}, \quad b_{7}=\chi_{s}
\end{gathered}
$$




\section{A Spinning Rayleigh Beam Under Pulsating Axial Load}

As a numerical example, we consider a Rayleigh beam with circular cross section spinning with constant speed $\Omega$. The beam is under axial load $P_{0}+P \cos \vartheta t$, where $\vartheta$ is the frequency of the dynamic loading. The dimensionless equation of motion can be written as

$$
\begin{aligned}
& \left(1-r^{*} \frac{\partial^{2}}{\partial x^{* 2}}\right) \frac{\partial^{2} v^{*}}{\partial t^{* 2}}+\left(2 i \Omega^{*} \frac{\partial^{2}}{\partial x^{* 2}}\right) \frac{\partial v^{*}}{\partial t^{*}} \\
& +\left[\frac{\partial^{4}}{\partial x^{* 4}}+\left(P_{0}^{*}+2 \epsilon \cos \vartheta^{*} t^{*}\right) \frac{\partial^{2}}{\partial x^{* 2}}\right] v^{*}=0
\end{aligned}
$$

where

$$
\begin{gathered}
x^{*}=\frac{x}{l}, \quad v^{*}=\frac{v}{l}, \quad t^{*}=\frac{t}{l^{2}} \sqrt{\frac{E I}{\rho A}}, \quad \Omega^{*}=\Omega \sqrt{\frac{\rho I}{E A}} \\
\vartheta^{*}=\vartheta l^{2} \sqrt{\frac{\rho A}{E I}}, \quad r^{*}=\frac{I}{l^{2} A}, \quad P_{0}^{*}=\frac{P_{0} l^{2}}{E I}, \quad 2 \epsilon=\frac{P l^{2}}{E I}
\end{gathered}
$$

$\rho$ is the mass density, $E$ is Young's modulus, $A$ and $I$ are the area and the moment of inertia of the cross section, and $l$ is the length of the beam. Complex displacement $v$ is defined as $v=$ $v_{1}+i v_{2}$, where $v_{1}$ and $v_{2}$ are two orthogonal displacements relative to the inertial frame. We assume that the beam is clamped at $x=0$ and is pinned at $x=1$. In the following discussion we neglect all asterisks for brevity.

We consider the case when the Rayleigh beam is under axial preload and parametric excitation $0.4 P_{c}+P \cos \vartheta t$, where $P_{c}$ $=20.19$ is the first buckling load of the non-spinning beam. In the case when the beam is not spinning, the lowest three natural frequencies are $\omega_{1}=11.96, \omega_{2}=45.83$, and $\omega_{3}=98.03$. When the beam spins at $\Omega=0.023$, these three modes split into six modes with natural frequencies $\omega_{1 f}=12.23, \omega_{1 b}=11.70, \omega_{2 f}$ $=46.79, \omega_{2 b}=44.89, \omega_{3 f}=100.07$, and $\omega_{3 b}=96.02$. The subscripts $f$ and $b$ represent forward and backward traveling
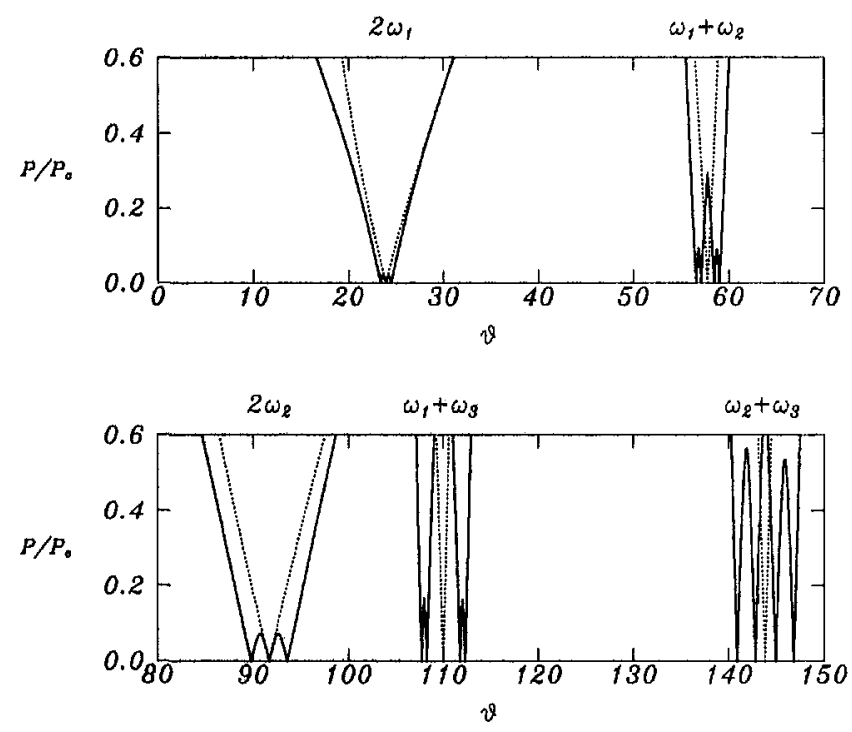

Fig. 1 Solid lines represent the transition curves for a clamped-pinned Rayleigh beam spinning at $\Omega=0.023$ and under parametric excitation $0.4 P_{c}+P \cos \vartheta t$. Dashed lines represent the case for a non-spinning beam.

modes, respectively. The solid lines in Fig. 1 are the transition curves up to the second order for the spinning beam in the $P \vartheta$ plane. The dashed lines representing the transition curves for the non-spinning beam are also shown for comparison. It is obvious from this figure that the unstable regions are in general wider for the spinning beam, primarily due to the frequency splitting. More specifically, when the non-spinning beam undergoes single mode resonance at the excitation frequency $\vartheta=$ $2 \omega_{1}$, there exist three subregions of instability for the spinning beam, i.e., $\vartheta=2 \omega_{1 f}, 2 \omega_{1 b}$, and $\omega_{1 f}+\omega_{1 b}$. When the nonspinning beam undergoes combination resonance at the excitation frequency $\vartheta=\omega_{1}+\omega_{2}$, there exist four subregions of instability for the spinning beam, i.e., $\vartheta=\omega_{1 f}+\omega_{2 f}, \omega_{1 f}+\omega_{2 b}$, $\omega_{1 b}+\omega_{2 f}$, and $\omega_{1 b}+\omega_{2 b}$.

\section{References}

Chen, J.-S., and Bogy, D. B., 1992, "Mathematical Structure of Modal Interactions in a Spinning Disk-Stationary Load System," ASME Journal of Applied Mechanics, Vol. 59, No. 2, Part 1, pp. 390-397.

Nayfeh, A. H., and Mook, D. T., 1977, "Parametric Excitations of Linear Systems Having Many Degrees of Freedom," Journal of Acoustical Society of America, Vol. 62, pp. 375-381. 